\title{
Comparative Meta-analysis of Adipose Tissue Transcriptomics Data in PCOS Patients and Healthy Controls Women
}

Saman Saedi ( $\nabla$ saman-saedi@shirazu.ac.ir)

Shiraz University https://orcid.org/0000-0002-9890-4934

Reza Panahi

Shiraz University

Najmeh Orak

Ahvaz Jundishapur University of Medical Sciences: Ahvaz Jondishapour University of Medical Sciences

Mohammad Reza Jafarzadeh Shirazi

Shiraz University

\section{Research Article}

Keywords: Adipose tissue, DEGs, KEGG analysis, Meta-analysis, PCOS

Posted Date: January 25th, 2022

DOI: https://doi.org/10.21203/rs.3.rs-1258689/v1

License: (c) This work is licensed under a Creative Commons Attribution 4.0 International License.

Read Full License 


\section{Abstract}

Background Women with polycystic ovary syndrome (PCOS) seem to have disturbances in lipid metabolism in subcutaneous adipose tissue. Nevertheless, gene expression in subcutaneous adipose tissue of PCOS women and its relation to other disturbances have been fragmentarily investigated.

Methods and results We utilized microarray data to identify the most important up and down-regulated candidate genes in adipose tissue of PCOS women in contrast to healthy women using the meta-analysis technique. Microarray data produced from three independent experiments $(n=3)$ conducted on adipose tissue in women with PCOS were retrieved from ArrayExpress. Then, the datasets were merged using the metaSeq package in Rstudio and differentially expressed genes (DEGs) in studies were selected. The integrative bioinformatics analyses of candidates were performed by gene ontology (GO) analysis, Kyoto Encyclopedia of Genes and Genomes (KEGG) analysis, and Protein-protein interaction (PPI) network construction. Twelve up-regulated genes and twelve down-regulated genes were identified and assessed as the most important DEGs. These the most important DEGs that were annotated by KEGG analysis were mainly involved in PI3K-Akt signaling pathway, pathways in cancer, breast cancer, metabolic pathways, ovarian steroidogenesis, and insulin secretion. In the PPI networks, PRDM10, FGFR2, IGF1R, and FLT1 were the key nodes in the up-regulated networks, while the NDUFAB1 and NME2 proteins were key in the down-regulated networks.

Conclusion Overall, these findings provide a comprehensive system biology based insight into the gene expression in subcutaneous adipose tissue of PCOS women and its relation to other disturbances.

\section{Introduction}

Polycystic ovary syndrome (PCOS) is the most common hyperandrogenic disorder affecting $5-8 \%$ of women of reproductive age [1]. PCOS is considered the foremost of ovarian diseases and usually diagnosed during the early reproductive years. Most women with PCOS display some metabolic abnormalities including dyslipidemia, obesity, hyperinsulinemia, neuroendocrine abnormalities, insulin resistance, and ovulatory dysfunction $[28,47]$. PCOS is considered a multifactorial disorder with various metabolic, genetic, endocrine, and environmental abnormalities [17].

Obesity, a characteristic of $60-80 \%$ of PCOS patients and plays a very important role in the development of PCOS in many women. Obesity alters levels of several hormones such as insulin, androgens, and adipocytokines [12]. This suggests that obesity modifies PCOS characteristics. It has been shown that obesity has a malignant additive effect on features of PCOS such as hyperandrogenism, insulin resistance, menstrual irregularity, ovulatory disorders, and pregnancy complications $[12,20]$.

Hyperandrogenism or increase in secretion of androgens can induce low-grade chronic inflammation by increasing the transcription of androgen receptors in mononuclear cells [51]. Low-grade chronic inflammation itself leads to the release of adipocytokines by dysfunctional adipocytes [2]. The role of adipocyte dysfunction in women with PCOS has very importance. 
Visceral adipose tissue is thought to be important for the pathogenesis of PCOS, because of its association with hyperandrogenemia and its often excessive accumulation in women with PCOS [3]. Increase in subcutaneous adipocytes and decrease in the secretion of adiponectin are major factors that strongly associated with insulin resistance [32]. Furthermore, changed adipose tissue expression of some genes such as TWIST1, CCL2, LEPR, and PPARG that may be important in the pathophysiology of PCOS have been reported $[5,7,10,23,45,46]$. Therefore, these findings indicate the adipose tissue dysfunction may negatively affect the metabolic health of women with PCOS and thereby increase their risk for diabetes mellitus type 2 (DM2), hyperandrogenism, and cardiovascular disease [31].

A recent meta-analysis from five comparative studies demonstrated that women with PCOS were three times more likely to develop endometrial cancer than healthy women [21]. Moreover, Gene expression profiles in subcutaneous fat from no obese women with and without PCOS disclosed differences in the expression of genes encoding components of several biological pathways related to insulin and Wnt signaling, lipid metabolism, immune function, inflammation, and oxidative stress [7, 10]. By the fact that dysfunctional adipose tissue is increasingly considered to be important in the metabolic disorders in patients with PCOS, in the present study, we utilized microarray data to identify the most important up and down-regulated candidate genes in adipose tissue in women with PCOS in contrast to healthy women using meta-analysis technique that is an attempt to integrate multiple data in different studies.

\section{Material And Methods}

\section{Microarray data}

To identify key up and down-regulated candidate genes in adipose tissue in women with PCOS in contrast to healthy women (control), microarray data produced from three independent experiments $(n=3)$ conducted on adipose tissue in women with PCOS were retrieved from ArrayExpress

(https://www.ebi.ac.uk/arrayexpress/). In all used experiments fat biopsy samples obtained from morbidly obese women with or without PCOS. In the first experiment (E-GEOD-43322) which was a casecontrol study we just exploited the data of 23 samples (sixteen PCOS patients and seven control). In the second experiment (E-GEOD-43264), we exploited the data of 15 samples (eight PCOS patients and seven control). In the third experiment (E-GEOD-5090), we exploited the data of 17 samples (nine PCOS patients which submitted to bariatric surgery because of morbid obesity and eight control) (Supplementary Table S1). The overall scheme of data analysis and computational tools used in this study is represented in Fig. 1.

\section{Meta-analysis}

Meta-analysis is an attempt to integrate multiple data in different studies. Indeed, by meta-analysis, genes which differentially expressed in many studies are selected as DEGs by metaSeq package [48]. Microarray studies were processed separately as individual datasets with FlexArray software version 1.6.3. The raw data were normalized using Robust Multiarray Average (RMA) algorithm and then RMA signal values were transformed into log2. Then, the datasets were merged using the metaSeq package in 
Rstudio and differentially expressed genes (DEGs) in studies were selected. To select DEGs, P-value was adjusted in false discovery rate (FDR) as less than $<0.05$, and list of genes was obtained for adipose tissue in women with PCOS in contrast to control. Venn diagram of all genes in three studies were generated by (http://bioinformatics.psb.ugent.be/webtools/Venn/).

\section{GO analysis and KEGG pathway enrichment analysis}

To gene ontology (GO) analysis, genes that were the most important up and down-regulated genes in adipose tissue from women with PCOS in contrast to control in three studies selected and were conducted using Gene Ontology Consortium tools (http://www.geneontology.org/) with default significance levels (less than $<0.05$ ). Then, the results were described in the forms of biological process, molecular function and cellular components [19]. In addition, KEGG (Kyoto Encyclopedia of Genes and Genomes) pathway enrichment analysis [25] was performed to identify the important pathways of the most important up and down-regulated genes in adipose tissue in women with PCOS. For this target, KEGG database (http://kobas.cbi.pku.edu.cn/) was used to test the enrichment of DGEs in KEGG pathways.

\section{Protein-protein interaction (PPI) network analysis}

Interaction protein networks for the mentioned up and down-regulated genes in adipose tissue in women with PCOS in contrast to control were constructed using the STRING v.10 (Search Tool for the Retrieval of Interacting Genes/Proteins) for detect functional association between those genes [11, 44]; this database is based on specific relationships between proteins and constructs associations based on distinct lines of evidence: Experimental evidence from PPI assays; recurring neighborhood of the genes in known genomes; co-expression databased on the expression data; events of fusion between those genes and a large collection of full-text articles [44].

\section{Results}

This study was conducted to identify key up and down-regulated candidate genes and obtain more information about these gens in adipose tissue in women with PCOS in contrast to healthy women.

This experiment was implemented based on microarray data retrieved from three independent studies. The results illustrated that the study of one have 3233 DEGs, whereas DEGs in studies of two and three were 1304 and 571 genes, respectively (Fig. 2). As a result some genes were common in adipose tissue from women in three studies (Fig. 2) Number of common genes that were significantly up and downregulated in adipose tissue in women with PCOS in contrast to healthy women was demonstrated in Fig. 2. Additionally, the most important DEGs in adipose tissue in women with PCOS in contrast to control women represented in Table 1. 
Table 1

The most important DEGs in adipose tissue in women with PCOS in contrast to control women.

\begin{tabular}{|c|c|c|c|c|}
\hline Ensembl ID & $\begin{array}{l}\text { Gene } \\
\text { symbol }\end{array}$ & Gene name & $\begin{array}{l}\text { log2 fold } \\
\text { change }\end{array}$ & FDR \\
\hline ENSG00000066468 & FGFR2 & Fibroblast growth factor receptor 2 & 4.67321 & 0.000421 \\
\hline ENSG00000077232 & DNAJC10 & $\begin{array}{l}\text { DnaJ homolog subfamily } \mathrm{C} \text { member } \\
10\end{array}$ & 4.16549 & 0.000584 \\
\hline ENSG00000138668 & $H N R N P D$ & $\begin{array}{l}\text { Heterogeneous nuclear } \\
\text { ribonucleoprotein DO }\end{array}$ & 3.76184 & 0.000761 \\
\hline ENSG00000170325 & PRDM10 & PR domain zinc finger protein 10 & 3.62374 & 0.000915 \\
\hline ENSG00000140443 & IGF1R & Insulin-like growth factor 1 receptor & 3.49823 & 0.002893 \\
\hline ENSG00000091879 & ANGPT2 & Angiopoietin-2 & 3.16138 & 0.007374 \\
\hline ENSG00000102755 & FLT1 & FMS-like tyrosine kinase & 3.01057 & 0.013101 \\
\hline ENSG00000109458 & $G A B 1$ & GRB2-associated-binding protein 1 & 2.97861 & 0.024932 \\
\hline ENSG00000069869 & NEDD4 & E3 ubiquitin-protein ligase NEDD4 & 2.83794 & 0.025716 \\
\hline ENSG00000204490 & $T N F-a$ & Tumor necrosis factor-alpha & 2.68917 & 0.025927 \\
\hline \multirow[t]{2}{*}{ ENSG00000101333 } & PLCB4 & $\begin{array}{l}\text { 1-phosphatidylinositol 4,5- } \\
\text { bisphosphate }\end{array}$ & 2.66108 & 0.027457 \\
\hline & & phosphodiesterase beta- 4 & & \\
\hline ENSG00000120868 & APAF1 & $\begin{array}{l}\text { Apoptotic protease-activating factor } \\
1\end{array}$ & 2.37294 & 0.027933 \\
\hline ENSG00000088832 & FKBP1A & $\begin{array}{l}\text { Peptidyl-prolyl cis-trans isomerase } \\
\text { FKBP1A }\end{array}$ & -5.30816 & 0.000035 \\
\hline ENSG00000243678 & NME2 & Nucleoside diphosphate kinase B & -4.84912 & 0.000074 \\
\hline ENSG00000168032 & ENTPD3 & $\begin{array}{l}\text { Ectonucleoside triphosphate } \\
\text { diphosphohydrolase } 3\end{array}$ & -4.75045 & 0.000238 \\
\hline ENSG00000078295 & $A D C Y 2$ & Adenylate cyclase type 2 & -4.53719 & 0.000641 \\
\hline ENSG00000004779 & NDUFAB1 & Acyl carrier protein, mitochondrial & -4.46018 & 0.000962 \\
\hline ENSG00000082515 & MRPL22 & $\begin{array}{l}39 S \text { ribosomal protein } \mathrm{L} 22 \text {, } \\
\text { mitochondrial }\end{array}$ & -4.06421 & 0.002856 \\
\hline ENSG00000180424 & $D E F B 1$ & Beta-defensin 1 & -3.75248 & 0.006187 \\
\hline ENSG00000189223 & $\begin{array}{l}\text { PAX8- } \\
\text { AS1 }\end{array}$ & Paired-box gene 8 & -3.70671 & 0.007413 \\
\hline ENSG00000164308 & ERAP2 & $\begin{array}{l}\text { Endoplasmic reticulum } \\
\text { aminopeptidase } 2\end{array}$ & -3.59318 & 0.007682 \\
\hline
\end{tabular}




\begin{tabular}{|lllll|}
\hline Ensembl ID & $\begin{array}{l}\text { Gene } \\
\text { symbol }\end{array}$ & Gene name & $\begin{array}{l}\text { log2 fold } \\
\text { change }\end{array}$ & FDR \\
\hline ENSG00000145777 & TSLP & Thymic stromal lymphopoietin & -3.16270 & 0.009814 \\
\hline ENSG00000163106 & HPGDS & $\begin{array}{l}\text { Hematopoietic prostaglandin D } \\
\text { synthase }\end{array}$ & -2.86374 & 0.023781 \\
\hline ENSG00000177868 & SVBP & Small vasohibin-binding protein & -2.79582 & 0.031863 \\
\hline
\end{tabular}

\section{Visualization of DEG position}

The genomic position of identified DEG on all chromosomes of human is represented in Fig. 3. Genomic position visualization was utilized for displaying the position of identified DEG in three studies on all human chromosomes. Accordingly, the highest number of DEG in the three studies were on 1 and 2 chromosomes.

\section{Functional classification of DEGs}

GO analysis was conducted to investigate other possible functions of the DEGs detected in the adipose tissue of women with PCOS in three studies. Accordingly, comparison of GO distribution of the DEGs indicated three distinct categories: the biological process (BP), molecular function (MF) and cellular component (CC). Up-regulated genes were enriched in $95 \mathrm{GO}$ functions $(P<0.05$, Fig. 4$)$. Of the upregulated genes, enrichment was mainly involved in the following BP: regulation of transcription, DNAtemplated, regulation of RNA biosynthetic process, regulation of gene expression, cellular nitrogen compound metabolic process, cellular aromatic compound metabolic process, and regulation of biological process, following MF: RNA binding, organic cyclic compound binding, and cation binding, and following CC: nucleoplasm part, nucleoplasm, membrane-enclosed lumen, and membrane-bounded organelle. Moreover, downregulated genes were enriched in $276 \mathrm{GO}$ functions $(P<0.05$, Fig. 4). Of the dow-nregulated genes, enrichment was mainly involved in the following BP: nucleoside monophosphate metabolic process, peptide metabolic process, immune response, intracellular transport, and organic substance metabolic process, following MF: RNA polymerase II transcription factor binding, histone binding, RNA polymerase II regulatory region sequence-specific DNA binding, DNA-binding transcription factor activity, and following CC: mitochondrial respiratory chain complex I, NADH dehydrogenase complex, respiratory chain complex I, ribosomal subunit (Supplementary Table S2).

\section{KEGG enrichment analysis of the most important DEGs}

KEGG enrichment analysis of the most important DEG targets revealed several pathways to gain greater perception into mechanisms of DEG biological functions in the adipose tissue of women with PCOS ( $P$ $<0.05$, Fig. 5). KEGG enrichment analysis demonstrated that the most important up-regulated expression genes were assigned into 20 pathways where the largest categories interestingly were involved in Ras signaling pathway, EGFR tyrosine kinase inhibitor resistance, MAPK signaling pathway, PI3K-Akt 
signaling pathway, pathways in cancer, cancers of the digestive system, long-term depression, gastric cancer, hepatocellular carcinoma, cancers of the lung and pleura, and hepatocellular carcinoma (Fig. 5). In contrast, the most important dow-nregulated gene expression was assigned into 7 pathways where the largest categories were involved in the metabolic pathways, pyrimidine metabolism, ovarian steroidogenesis, insulin secretion, and GnRH signaling pathway (Fig. 5). The complete significant KEGG pathways for the most important DEGs are provided in Supplementary Table S3.

\section{Analysis of the PPI networks of the most important DEGs}

To explore the regulation mechanism of key DEGs in the adipose tissue of women with PCOS, the most important DEGs were imputed into STRING to construct a PPI network, and the network was visualized for up and down-regulated genes separately (Fig. 6 and 7). In PPI Networks, the most important up and down-regulated genes with the highest hub scores were identified as hub genes that highly correlated with these pathways-related genes, suggesting their regulatory function (Fig. 6 and 7). Hub genes play a determinant role in gene regulation because of their central position in the network. In the PPI networks of DEGs, PRDM10, FGFR2, IGF1R, and FLT1 were the key nodes in the up-regulated networks (Fig. 6), while the NDUFAB1 and NME2 proteins were key in the down-regulated networks (Fig. 7).

\section{Discussion}

Given the central role of adipose tissue in the development of PCOS in overweight and obese women, this comparative meta-analysis was performed to show changes in the expression patterns of genes between adipose tissue of PCOS women and control samples. Our result showed changes in the expression patterns of several genes in adipose tissue of PCOS women and its relation to future possible disturbances. In our meta-analysis study, the majority of significantly enriched pathways are involved in immune diseases, cancer, and insulin secretion emphasizing a critical role in the pathogenesis of PCOS.

In this study, we found that TNF-a and ANGPT2 genes were dramatically up-regulated in the adipose tissue of PCOS women in contrast to healthy women (Table 1). The role of these up-regulated genes has been demonstrated in pathogenesis of PCOS and cancer. Tumor necrosis factor a (TNF- $a$ ) is a proinflammatory cytokine that is thought to play a role in the pathogenesis of PCOS [35]. Although TNF-a is involved in adipocyte metabolism [6], it has been reported that mRNA expression of TNF-a is similar levels in adipose tissue of women with and without PCOS [31]. Meanwhile, TNF-a causes insulin resistance in adipose tissue [6], and may affect the onset of type 2 diabetes mellitus (T2DM) [43]. T2DM and insulin resistance stimulate ovarian and adrenal androgen production and lead to PCOS [13]. Although the ovaries are the main source of increased androgen (hyperandrogenism) in PCOS [16], adrenal androgen excess can be present in approximately $20-25 \%$ among women with PCOS [15]. Thus, hyperandrogenism has a multifactorial origin that overexpression of TNF-a also is one of them. Of note, overexpression of TNF-a can trigger the delivery of Angiopoietin-2 (ANGPT2) into the blood [41]. ANGPT2 is a growth factor regulating vessel growth and maturation during angiogenesis [37]. ANGPT2 is expressed by activated endothelial cells under usual conditions, can also be produced by mesenchymal stem cells and by tumor cells in hypoxia and cancers conditions. In addition to its proangiogenic role 
during cancer progression, ANGPT2 contributes to metastatic formation [40]. It has been previously that ANGPT2 is a prognostic factor in localized metastatic colorectal cancer (CRC) [22]. Therefore, upregulation of ANGPT2 in adipose tissue of PCOS women might be considered as a biomarker in the early detection of cancer.

Our meta-analysis result showed that the expression of HPGDS and TSLP genes were dramatically downregulated in the adipose tissue from women with PCOS (Table 1). HPGDS is a Sigma-class glutathione transferase expressed in peripheral tissues such as the placenta, intestine, and adipose tissue [24] and catalyzes the isomerization of prostaglandin $\mathrm{H} 2$ (PGH2) to prostaglandin D2 (PGD2) [50]. Inhibiting the production of PGD2 by inhibiting HPGDS may make it an interesting target to treat allergic inflammation [39]. HPGDS may play a role in the regulation of inflammation and epithelial cell health within the oviduct, an effect likely required to maintain homeostasis and function of this key reproductive organ [4]. In our meta-analysis study, HPGDS expression was down-regulated in adipose tissue from women with PCOS; however, investigation of HPGDS-regulated inflammation in PCOS disorder should be examined.

Another interesting gene among the most important down-regulated in adipose tissue in women with PCOS was thymic stromal lymphopoietin (TSLP), which acts as a co-stimulator for thymocyte proliferation [9]. Recent studies have been reported an expanding role for TSLP in inflammatory diseases and cancer [9]. Moreover, It has been found that overexpression of TSLP (in K14-TSLP transgenic mice) can inhibit the development of early breast cancer [14]. Expression of TSLP has been reported in visceral human adipose tissue $[30,49]$. However, the level of mRNA expression of TSLP is lower in obese women with metabolic syndrome (state of insulin resistance associated with central obesity) compared to those without metabolic syndrome [49]. The association of abdominal central obesity with insulin resistance and T2DM characterize many patients with PCOS [34]. Therefore, activation of TSLP signaling may be a therapeutic immunotarget for improving insulin sensitivity and preventing T2DM [8].

Of the most important DEG, two up-regulated genes (FGFR2 and IGF1R) in the adipose tissue of women with PCOS were the key nodes in the up-regulated PPI networks (Fig. 6). Interestingly, KEGG analysis results showed that these genes are related to pathways in cancer, MAPK signaling pathway, and PI3KAkt signaling pathway. Fibroblast growth factor receptor 2 (FGFR2) and insulin-like growth factor receptor (IGF1R) as specific receptor tyrosine kinases (RTKs) [38], which dysregulation of RTKs activity contributes to the progression of many cancers [27]. FGFR2 has a critical role in mammary development [29] and in the maintenance of breast tumor-initiating cells [26], resulting has been identified as a breast cancer risk [18]. Low expression of FGFR2 is associated with lower numbers of breast tumor-initiating cells [26]. IGF1R regulate androgen biosynthesis and involved in insulin secretion and action [36]. Dysregulation of the IGF-1/insulin/IGF-1R system may contribute to the pathophysiology of PCOS [33]. Therefore, overexpression of FGFR2 and IGF1R genes in the adipose tissue of women with PCOS may be associated with risk for breast cancer and PCOS.

In this meta-analysis study, KEGG analysis results showed that ADCY2 in the adipose tissue from women with PCOS was identified to play a potential role in metabolic pathways, ovarian steroidogenesis, insulin 
secretion, and GnRH signaling pathway. Adenylyl cyclase type 2 (ADCY2) encodes the adenylate cyclase, which can catalyze ATP to form the second messenger cyclic adenosine monophosphate (cAMP) [42]. Recently, has been reported that the expression of ADCY2 promote by estradiol (E2) [52]. Therefore, impaired E2 secretion in women with PCOS might decrease ADCY2 expression in the adipose tissue.

\section{Conclusion}

In conclusion, as subcutaneous adipose tissue is important for the pathogenesis of PCOS, this study provides more insight into the gene expression in subcutaneous adipose tissue of PCOS women and its relation to other disturbances. We showed that PCOS is associated with aberrant subcutaneous adipose tissue genes expression with dysregulated pathways including pathways in cancer, cancers of the digestive system, breast cancer, metabolic pathways, ovarian steroidogenesis, insulin secretion, and $\mathrm{GnRH}$ signaling pathway. This is a meta- analysis study and the cause-and-effect relationship was not clear; therefore, it is not possible to infer that PCOS alter the subcutaneous adipose tissue genes expression or contrariwise. Nevertheless, our findings need to be confirmed in prospective studies. Therefore, further studies are recommended.

\section{Declarations}

\section{Authors' contributions}

Authors' contributions: S. Saedi, R. Panahi, N. Orak, and M.R. Jafarzadeh Shirazi equally contributed to the conception and design of the research; S. Saedi, and R. Panahi, contributed to database search and collection of data; S. Saedi, R. Panahi, and M.R. Jafarzadeh Shirazi contributed to the acquisition and analysis of the data; S. Saedi, and N. Orak, contributed to the interpretation of the data; S. Saedi, and N. Orak contributed to draft the manuscript. All authors are in agreement with the manuscript and declare that there is no conflict of interest.

\section{Funding}

This study was conducted without external funding.

\section{Conflict of interest}

The authors do not have any conflict of interest to declare.

\section{Acknowledgments}

The authors would like to thank Bahman Khahani for his assistance in the interpretation of the data. 


\section{Supplementary Information}

The online version contains supplementary material available at

\section{Ethical approval}

This article does not contain any studies with human participants or animals performed by any of the authors.

\section{References}

1. Azziz R, Woods KS, Reyna R et al (2004) The prevalence and features of the polycystic ovary syndrome in an unselected population. The Journal of Clinical Endocrinology \& Metabolism 89:2745-2749

2. Baglioni S, Cantini G, Poli G et al (2012) Functional differences in visceral and subcutaneous fat pads originate from differences in the adipose stem cell. PLoS ONE 7:e36569

3. Barber T, Franks S (2013) Adipocyte biology in polycystic ovary syndrome. Mol Cell Endocrinol 373:68-76

4. Bridges PJ, Jeoung M, Shim S et al (2012) Hematopoetic prostaglandin D synthase: an ESR1dependent oviductal epithelial cell synthase. Endocrinology 153:1925-1935

5. Carmina E, Chu MC, Moran C et al (2008) Subcutaneous and omental fat expression of adiponectin and leptin in women with polycystic ovary syndrome. Fertil Steril 89:642-648

6. Cawthorn WP, Sethi JK (2008) TNF-a and adipocyte biology. FEBS Lett 582:117-131

7. Chazenbalk G, Chen Y-H, Heneidi S et al (2012) Abnormal expression of genes involved in inflammation, lipid metabolism, and Wnt signaling in the adipose tissue of polycystic ovary syndrome. The Journal of Clinical Endocrinology 97:E765-E770

8. Choa R, Wada S, Meng H et al (2020) The role of Thymic Stromal Lymphopoietin (TSLP) in protection from Type II diabetes. In:Am Assoc Immnol

9. Corren J, Ziegler SF (2019) TSLP: from allergy to cancer. Nat Immunol 20:1603-1609

10. Cortón M, Botella-Carretero JI, Benguria A et al (2007) Differential gene expression profile in omental adipose tissue in women with polycystic ovary syndrome. The Journal of Clinical Endocrinology \& Metabolism 92:328-337

11. De Abreu Neto JB, Frei M (2016) Microarray meta-analysis focused on the response of genes involved in redox homeostasis to diverse abiotic stresses in rice. Front Plant Sci 6:1260

12. De Medeiros SF, Rodgers RJ, Norman RJ (2021) Adipocyte and steroidogenic cell cross-talk in polycystic ovary syndrome. Human Reproduction Update

13. Delitala AP, Capobianco G, Delitala G et al (2017) Polycystic ovary syndrome, adipose tissue and metabolic syndrome. Arch Gynecol Obstet 296:405-419 
14. Demehri S, Cunningham TJ, Manivasagam S et al (2016) Thymic stromal lymphopoietin blocks early stages of breast carcinogenesis. J Clin Investig 126:1458-1470

15. Diamanti-Kandarakis E, Dunaif A (2012) Insulin resistance and the polycystic ovary syndrome revisited: an update on mechanisms and implications. Endocr Rev 33:981-1030

16. Ehrman DA, Barnes RB, Rosenfield RL (1995) Polycystic ovary syndrome as a form of functional ovarian hyperandrogenism due to dysregulation of androgen secretion. Endocr Rev 16:322-353

17. Emami N, Alizadeh A, Moini A et al (2020) Differences in fatty acid profiles and desaturation indices of abdominal subcutaneous adipose tissue between pregnant women with and without PCOS. Adipocyte 9:16-23

18. Fletcher MN, Castro MA, Wang X et al (2013) Master regulators of FGFR2 signalling and breast cancer risk. Nat Commun 4:1-12

19. Fruzangohar M, Ebrahimie E, Adelson DL (2014) Application of global transcriptome data in gene ontology classification and construction of a gene ontology interaction network. bioRxiv:004911

20. Galtier-Dereure F, Boegner C, Bringer J (2000) Obesity and pregnancy: complications and cost. Am J Clin Nutr 71:1242S-1248S

21. Haoula Z, Salman M, Atiomo W (2012) Evaluating the association between endometrial cancer and polycystic ovary syndrome. Hum Reprod 27:1327-1331

22. Jary M, Hasanova R, Vienot A et al (2020) Molecular description of ANGPT2 associated colorectal carcinoma. Int J Cancer 147:2007-2018

23. Jones MR, Chazenbalk G, Xu N et al (2012) Steroidogenic regulatory factor FOS is underexpressed in polycystic ovary syndrome (PCOS) adipose tissue and genetically associated with PCOS susceptibility. The Journal of Clinical Endocrinology \& Metabolism 97:E1750-E1757

24. Jowsey IR, Thomson AM, Flanagan JU et al (2001) Mammalian class Sigma glutathione Stransferases: catalytic properties and tissue-specific expression of human and rat GSH-dependent prostaglandin D2 synthases. Biochem J 359:507-516

25. Kanehisa M, Araki M, Goto S et al (2007) KEGG for linking genomes to life and the environment. Nucleic Acids Res 36:D480-D484

26. Kim S, Dubrovska A, Salamone RJ et al (2013) FGFR2 promotes breast tumorigenicity through maintenance of breast tumor-initiating cells. PLoS ONE 8:e51671

27. Krause DS, Van Etten RA (2005) Tyrosine kinases as targets for cancer therapy. N Engl J Med 353:172-187

28. Livadas S, Diamanti-Kandarakis E (2013) Polycystic ovary syndrome: definitions, phenotypes and diagnostic approach. Polycystic Ovary Syndrome 40:1-21

29. Lu P, Ewald AJ, Martin GR et al (2008) Genetic mosaic analysis reveals FGF receptor 2 function in terminal end buds during mammary gland branching morphogenesis. Dev Biol 321:77-87

30. Ma L, Zhen J, Sorisky A (2020) Regulators of thymic stromal lymphopoietin production by human adipocytes. Cytokine 136:155284 
31. Mannerås-Holm L, Benrick A, Stener-Victorin E (2014) Gene expression in subcutaneous adipose tissue differs in women with polycystic ovary syndrome and controls matched pair-wise for age, body weight, and body mass index. Adipocyte 3:190-196

32. Mannerås-Holm L, Leonhardt $\mathrm{H}$, Kullberg $\mathrm{J}$ et al (2011) Adipose tissue has aberrant morphology and function in PCOS: enlarged adipocytes and low serum adiponectin, but not circulating sex steroids, are strongly associated with insulin resistance. The Journal of Clinical Endocrinology \& Metabolism 96:E304-E311

33. Martin MBR, Witchel SF (2012) Growth and the Insulin-Like Growth Factor-1 Receptor (IGF1R). Handbook of growth and growth monitoring in health and disease. Springer, pp 2711-2722

34. Murri M, Insenser M, Escobar-Morreale HF (2014) Metabolomics in polycystic ovary syndrome. Clin Chim Acta 429:181-188

35. Ojeda-Ojeda M, Murri M, Insenser $M$ et al (2013) Mediators of low-grade chronic inflammation in polycystic ovary syndrome (PCOS). Curr Pharm Design 19:5775-5791

36. Prado Correia LE, De Almeida BC, De Jesus Simões M et al (2017) IGF-1R and leptin expression profile and the effects of metformin treatment on metabolic and endocrine parameters in PCOS mice. BioMed research international 2017

37. Reiss $Y$, Knedla A, Tal AO et al (2009) Switching of vascular phenotypes within a murine breast cancer model induced by angiopoietin-2. The Journal of Pathology: A Journal of the Pathological Society of Great Britain and Ireland 217:571-580

38. Rettew A, Young E, Lev D et al (2012) Multiple receptor tyrosine kinases promote the in vitro phenotype of metastatic human osteosarcoma cell lines. Oncogenesis 1:e34-e34

39. Rittchen S, Heinemann A (2019) Therapeutic potential of hematopoietic prostaglandin D2 synthase in allergic inflammation. Cells 8:619

40. Scholz A, Lang V, Henschler R et al (2011) Angiopoietin-2 promotes myeloid cell infiltration in a $\beta 2$ integrin-dependent manner. Blood The Journal of the American Society of Hematology 118:50505059

41. Scott BB, Zaratin PF, Colombo A et al (2002) Constitutive expression of angiopoietin-1 and-2 and modulation of their expression by inflammatory cytokines in rheumatoid arthritis synovial fibroblasts. J Rhuematol 29:230-239

42. Sethna F, Feng W, Ding Q et al (2017) Enhanced expression of ADCY1 underlies aberrant neuronal signalling and behaviour in a syndromic autism model. Nat Commun 8:1-11

43. Swaroop JJ, Rajarajeswari D, Naidu J (2012) Association of TNF-a with insulin resistance in type 2 diabetes mellitus. Indian J Med Res 135:127

44. Szklarczyk D, Franceschini A, Wyder S et al (2014) STRING v10: protein-protein interaction networks, integrated over the tree of life. Nucleic Acids Res 43:D447-D452

45. Tan B, Chen J, Digby J et al (2006) Upregulation of adiponectin receptor 1 and 2 mRNA and protein in adipose tissue and adipocytes in insulin-resistant women with polycystic ovary syndrome. Diabetologia 49:2723-2728

Page $12 / 19$ 
46. Tan BK, Adya R, Farhatullah S et al (2008) Omentin-1, a novel adipokine, is decreased in overweight insulin-resistant women with polycystic ovary syndrome: ex vivo and in vivo regulation of omentin-1 by insulin and glucose. Diabetes 57:801-808

47. Teede HJ, Hutchison S, Zoungas $S$ et al (2006) Insulin resistance, the metabolic syndrome, diabetes, and cardiovascular disease risk in women with PCOS. Endocrine 30:45-53

48. Tsuyuzaki K, Nikaido I (2013) metaSeq: Meta-analysis of RNA-seq count data. Tokyo University of Science, Tokyo

49. Turcot V, Bouchard L, Faucher $\mathrm{G}$ et al (2012) Thymic stromal lymphopoietin: an immune cytokine gene associated with the metabolic syndrome and blood pressure in severe obesity. Clin Sci 123:99109

50. Uchida Y, Urade Y, Mori S et al (2010) UV resonance Raman studies on the activation mechanism of human hematopoietic prostaglandin D2 synthase by a divalent cation. Mg2+ Journal of inorganic biochemistry 104:331-340

51. Yang R, Yang S, Li R et al (2016) Effects of hyperandrogenism on metabolic abnormalities in patients with polycystic ovary syndrome: a meta-analysis. Reproductive Biology and Endocrinology 14:1-10

52. Zhao G, Li X, Miao H et al (2020) Estrogen Promotes cAMP Production in Mesenchymal Stem Cells by Regulating ADCY2. International journal of stem cells 13:55

\section{Figures}

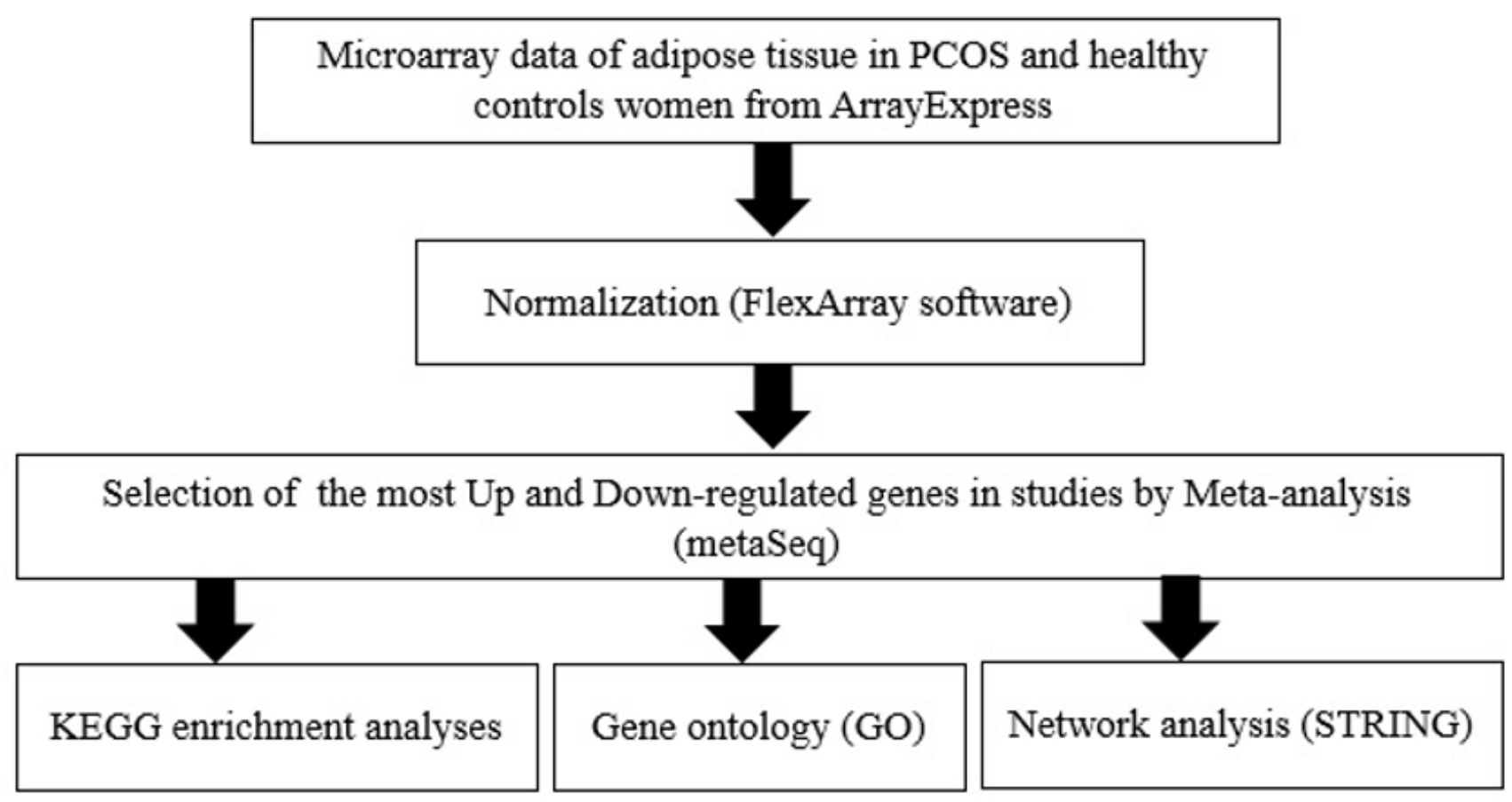

Figure 1 


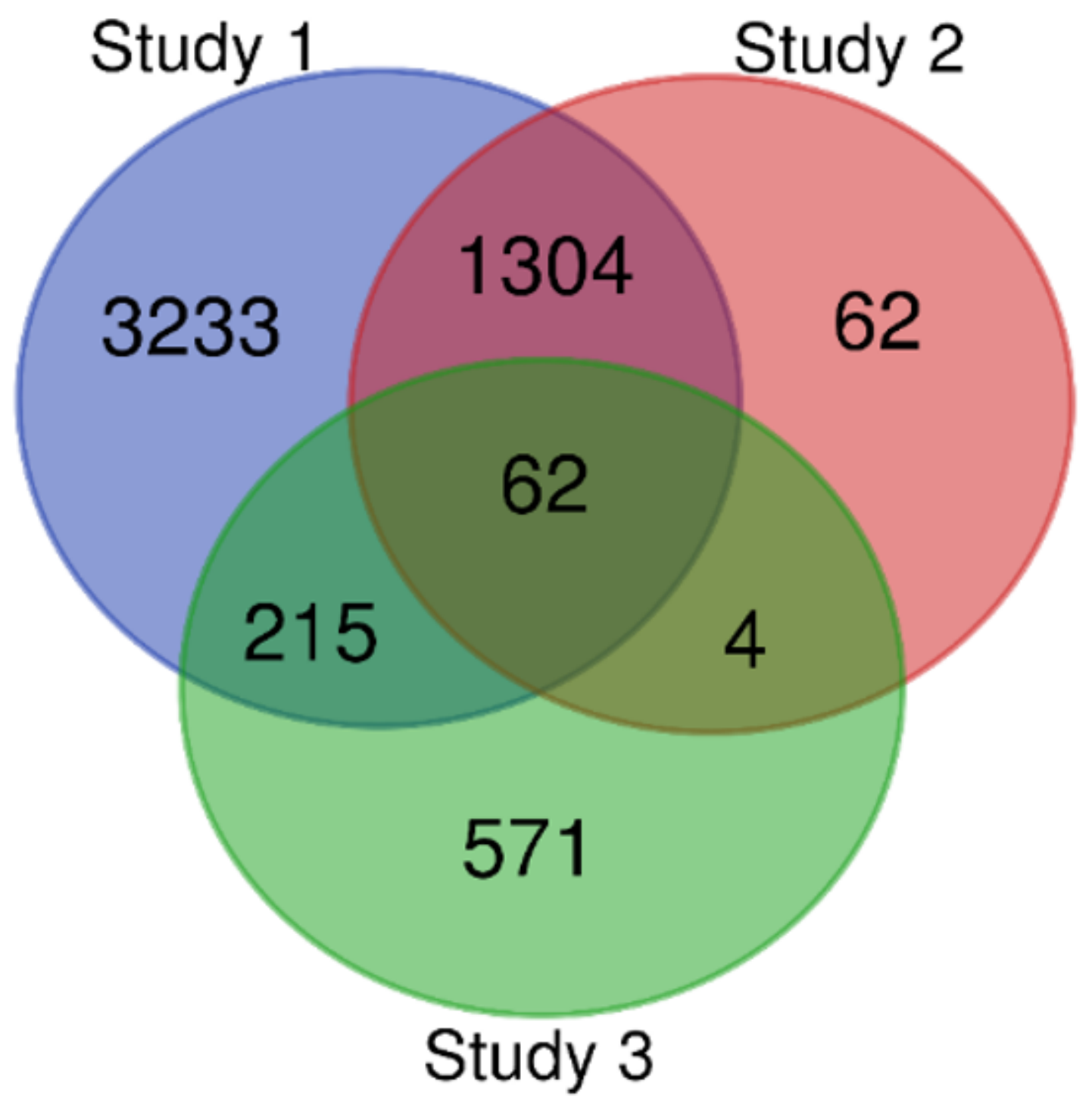

Figure 2

Venn diagrams of common differential expressed genes (DEG) in three studies through individual analysis based on FlexArray. 


\section{A}

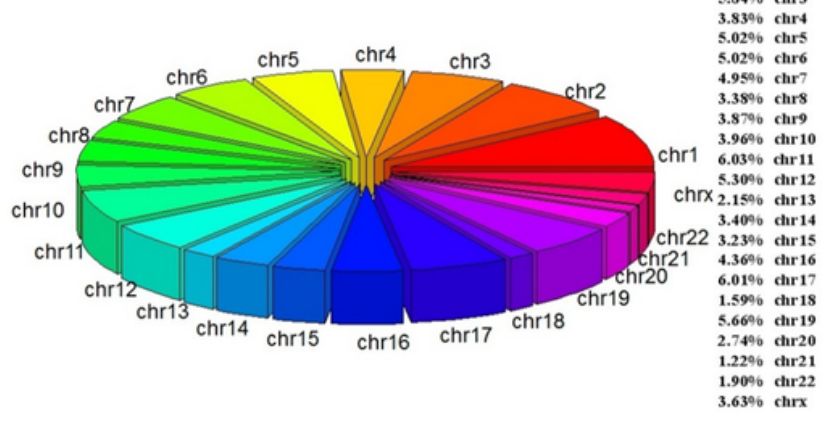

B

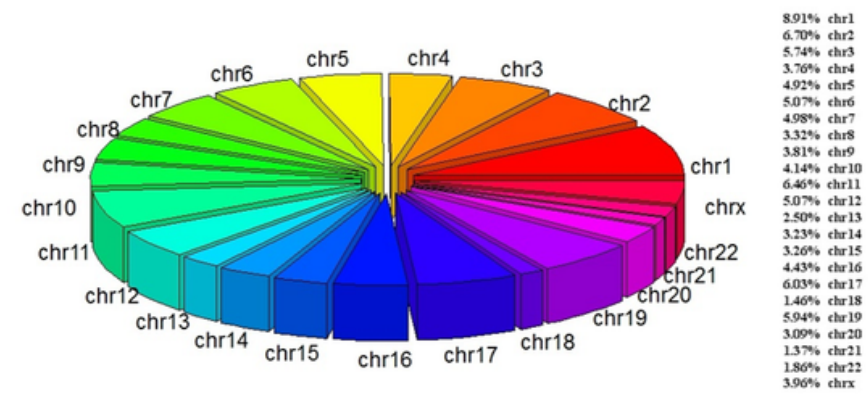

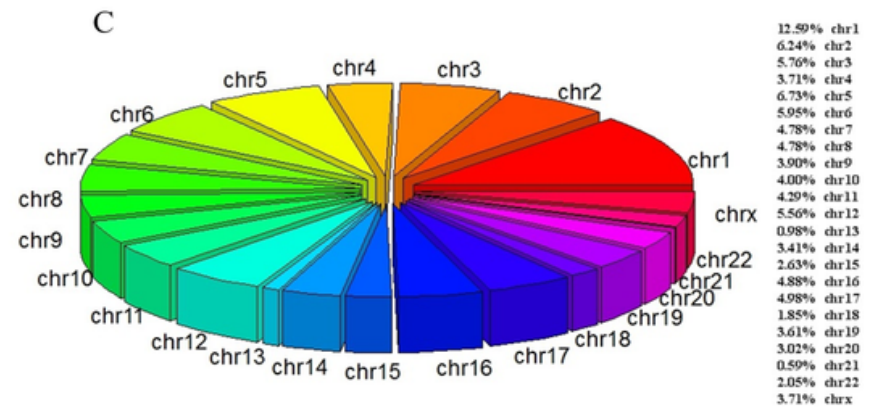

Figure 3

Schematic representation of the genomic position of DEG on all chromosomes of human. The first study (A), second study (B), and third study (C). 


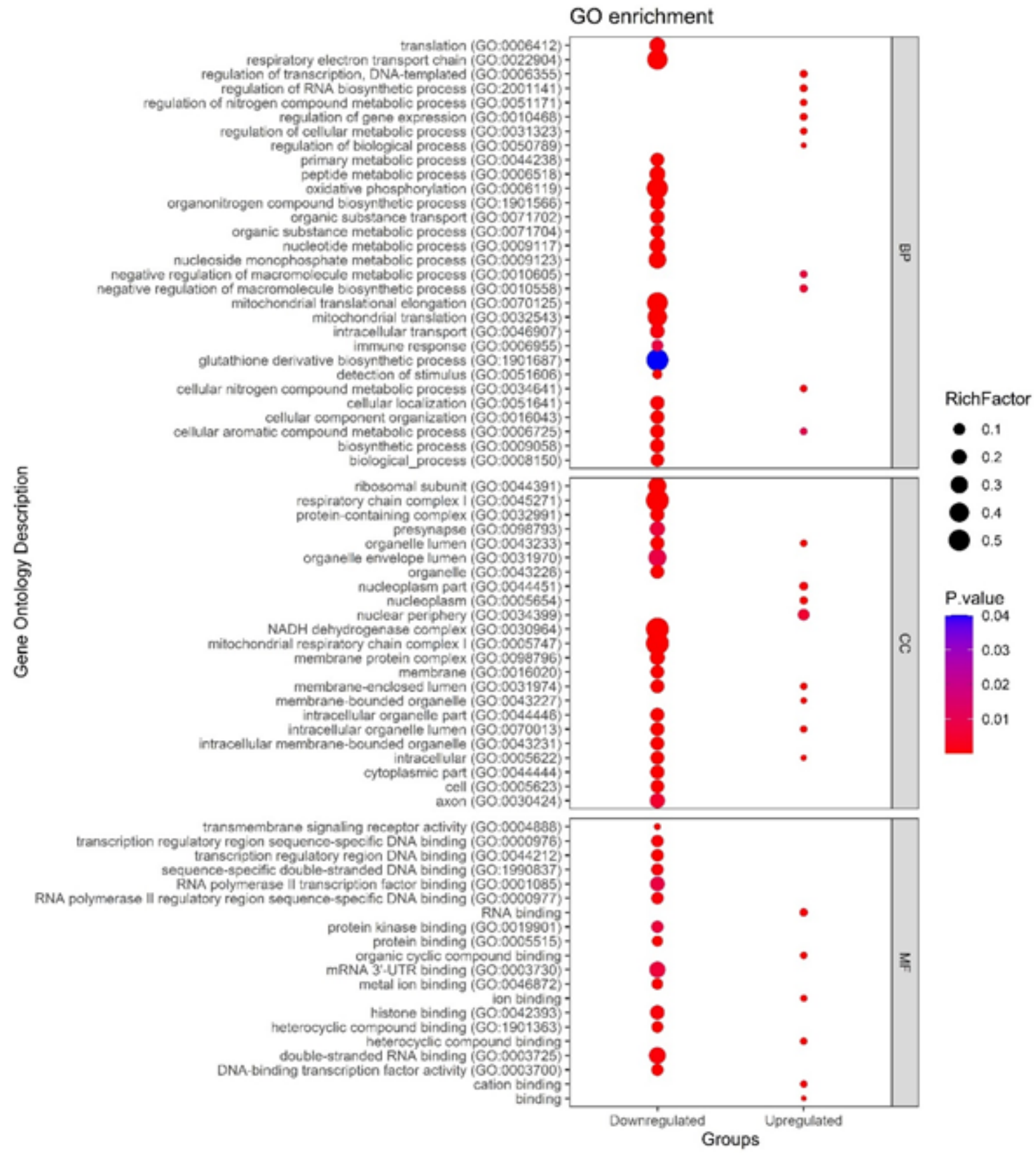

Figure 4

Functional category enrichment analysis of the DEGs in adipose tissue in women with PCOS in contrast to control women. Indicates significantly enriched gene ontology (G0) terms classify based on biological processes (BP), cellular components (CC), and molecular functions (MF). 


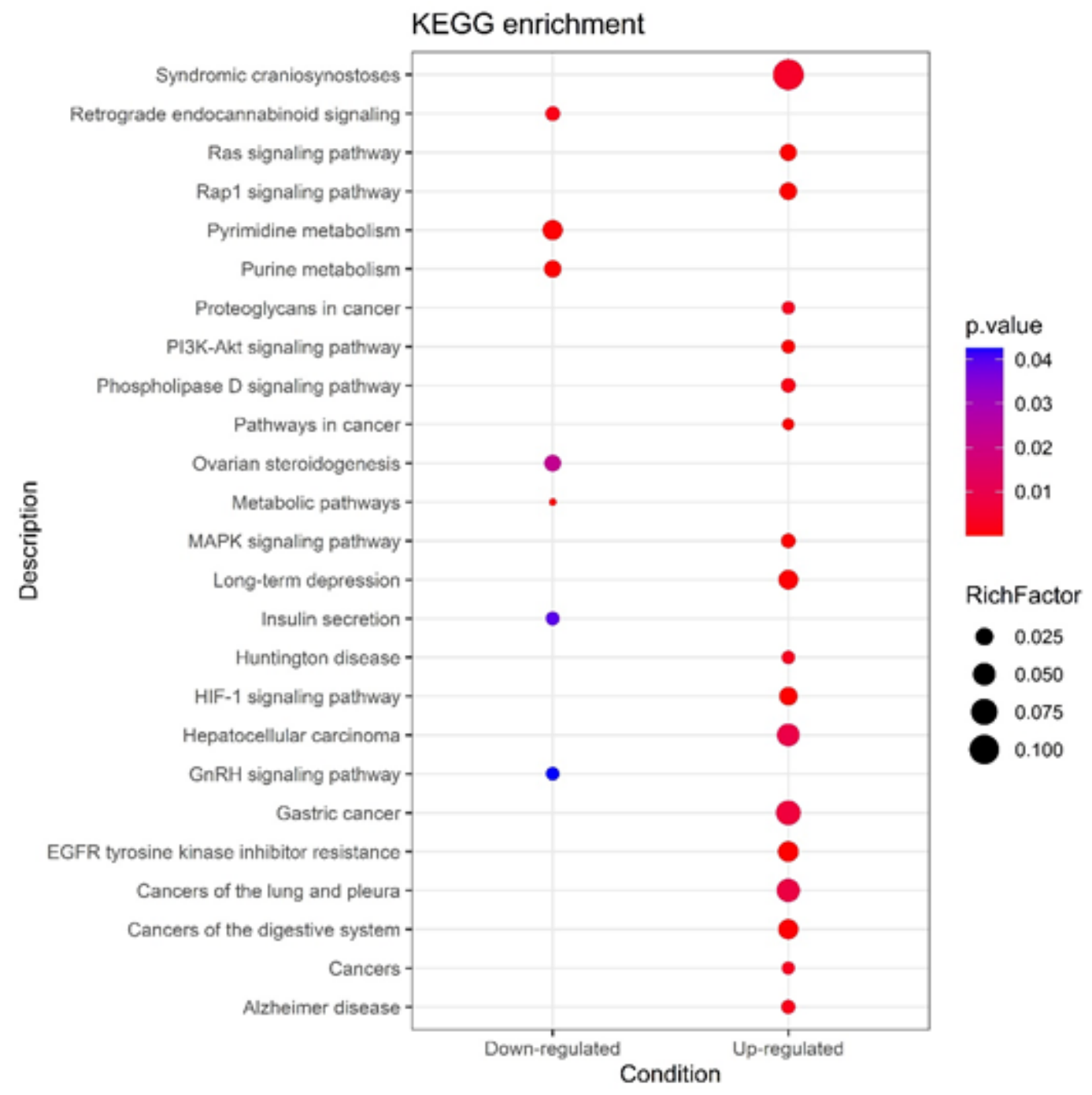

Figure 5

KEGG enrichment analyses of the most important DEGs in adipose tissue in women with PCOS in contrast to control women. The size of P-value is associated with each color in color scale bar and the size of dot reflects the number of DEGs in each pathway. 


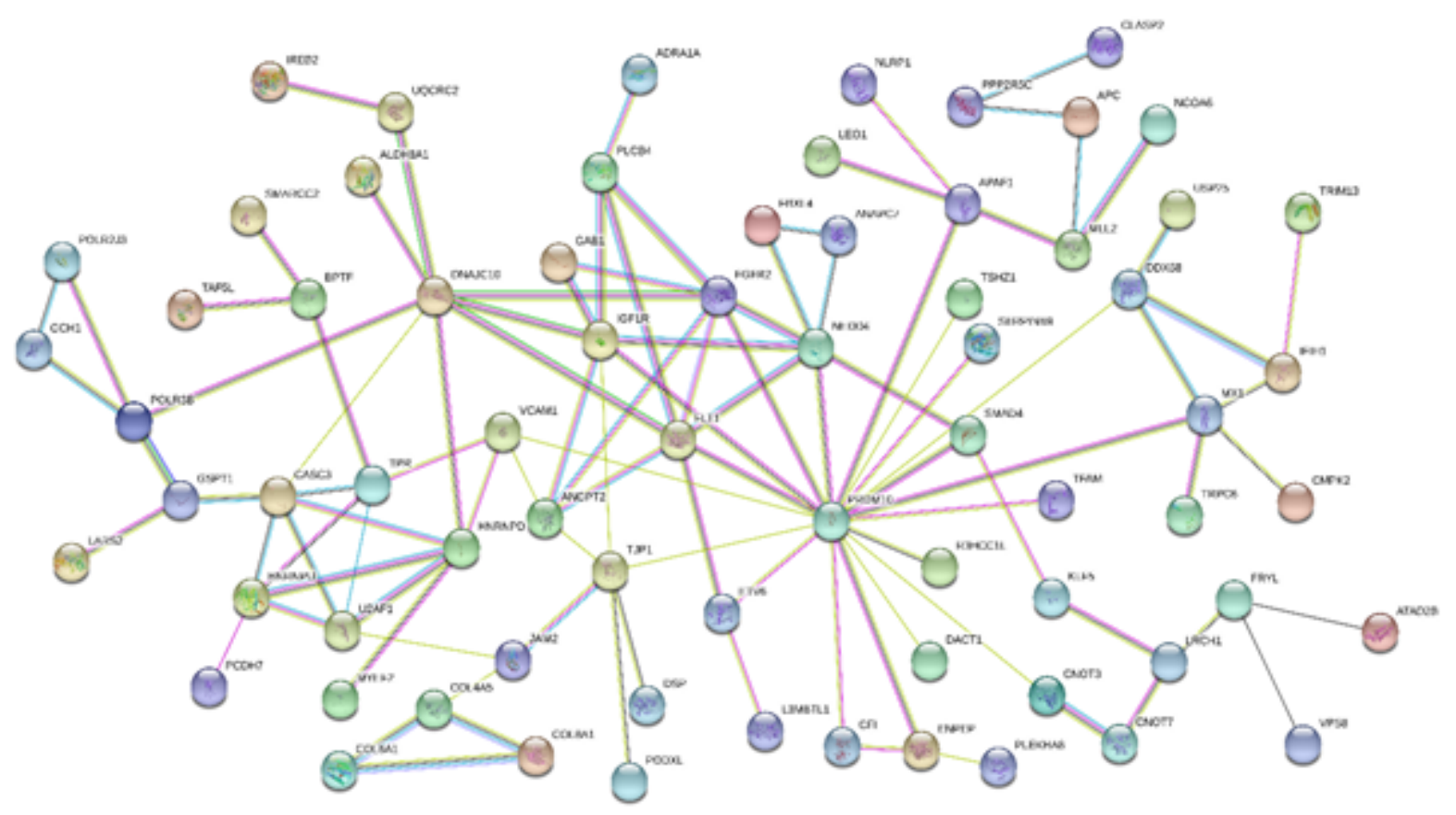

Figure 6

Gene network of the most important Up-regulated genes in adipose tissue in women with PCOS in contrast to control women. The connection difference colors represent the types of evidence for inferring association: recurring Neighborhood in different genomes (green line), Co-occurrence of those genes in the same organisms (dark blue), Experimental protein-protein interaction data (pink), events of Gene Fusion (red), co-expression (black), pathway described by other databases (light blue), literature textmining (yellow), and homology (purple lines). 


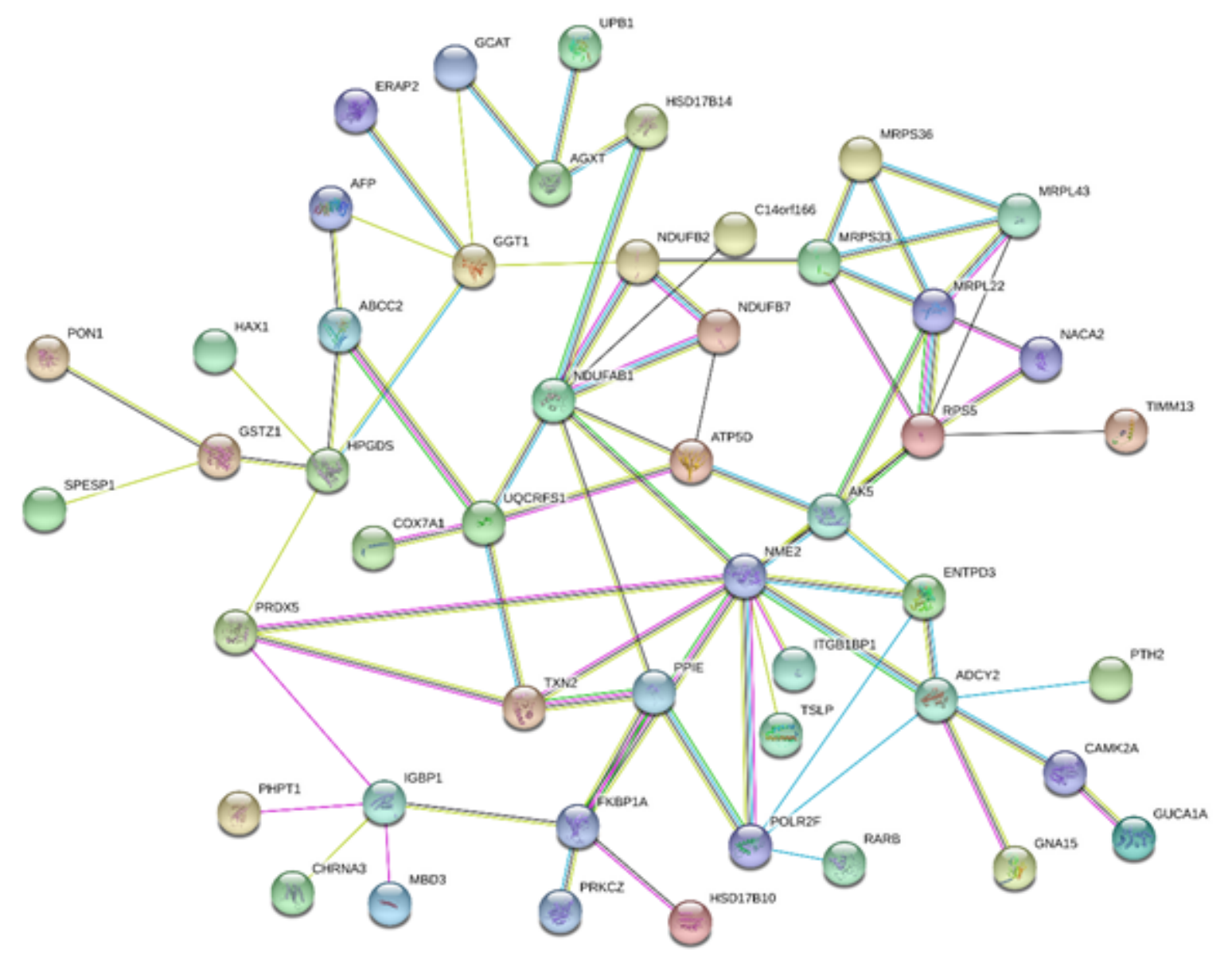

Figure 7

Gene network of the most important Down-regulated genes in adipose tissue in women with PCOS in contrast to control women. The connection difference colors represent the types of evidence for inferring association: recurring Neighborhood in different genomes (green line), Co-occurrence of those genes in the same organisms (dark blue), Experimental protein-protein interaction data (pink), events of Gene Fusion (red), co-expression (black), pathway described by other databases (light blue), literature textmining (yellow), and homology (purple lines).

\section{Supplementary Files}

This is a list of supplementary files associated with this preprint. Click to download.

- SupplementaryTableS1.xlsx

- SupplementaryTableS2.xlsx

- SupplementaryTableS3.xlsx 